\title{
Alzheimer's Disease Detection in Brain Magnetic Resonance Images Using Multiscale Fractal Analysis
}

\author{
Salim Lahmiri and Mounir Boukadoum \\ Department of Computer Science, University of Quebec at Montreal, 201 President-Kennedy, Local PK-4150, \\ Montreal, QC, Canada H2X $3 Y 7$ \\ Correspondence should be addressed to Mounir Boukadoum; boukadoum.mounir@uqam.ca
}

Received 15 July 2013; Accepted 19 September 2013

Academic Editors: P. A. Narayana and K. Tsuchiya

Copyright (C) 2013 S. Lahmiri and M. Boukadoum. This is an open access article distributed under the Creative Commons Attribution License, which permits unrestricted use, distribution, and reproduction in any medium, provided the original work is properly cited.

\begin{abstract}
We present a new automated system for the detection of brain magnetic resonance images (MRI) affected by Alzheimer's disease (AD). The MRI is analyzed by means of multiscale analysis (MSA) to obtain its fractals at six different scales. The extracted fractals are used as features to differentiate healthy brain MRI from those of AD by a support vector machine (SVM) classifier. The result of classifying 93 brain MRIs consisting of 51 images of healthy brains and 42 of brains affected by AD, using leave-one-out crossvalidation method, yielded $99.18 \% \pm 0.01$ classification accuracy, $100 \%$ sensitivity, and $98.20 \% \pm 0.02$ specificity. These results and a processing time of 5.64 seconds indicate that the proposed approach may be an efficient diagnostic aid for radiologists in the screening for $\mathrm{AD}$.
\end{abstract}

\section{Introduction}

Alzheimer's disease $(\mathrm{AD})$ is a progressive and degenerative disease that affects brain cells, and its early diagnosis has been essential for appropriate intervention by health professionals. Noninvasive in vivo neuroimaging techniques such as magnetic resonance imaging (MRI) and positron emission tomography (PET) are commonly used to diagnose and monitor the progression of the disease and the effect of treatment. In this regard, the problem of developing computer aided diagnosis (CAD) tools to distinguish images with $\mathrm{AD}$ from those of normal brains has been extensively addressed in the past years [1-12]. A review of a recent work follows.

Magnin et al. [1] used the relative weight of gray matter versus white matter and cerebrospinal fluid in 90 regions of interests (ROI) as features classified with SVM. Based on the bootstrap method, the SVM obtained $94.5 \%$ average classification accuracy in the classification of $16 \mathrm{AD}$ and 22 control (healthy) subjects, with a mean specificity of $96.6 \%$ and a mean sensitivity of $91.5 \%$. Ramírez et al. [2] proposed a classification system for AD based on the partial least square (PLS) regression model for feature extraction (identification of discriminative voxels) and the random forest (RF) classifier. The PLS-RF system yielded accuracy, sensitivity, and specificity values of $96.9 \%, 100 \%$, and $92.7 \%$, respectively, after classifying 41 normal and $56 \mathrm{AD}$ images using the leaveone-out cross-validation method. Salas-Gonzalez et al. [3] used Welch's $t$-test to identify voxels that provide higher difference between normal and $\mathrm{AD}$ images. The identified voxels formed the main features to classify, and a SVM with linear kernel reached $96.2 \%$ accuracy in distinguishing 41 normal and $38 \mathrm{AD}$ images using leave-one-out crossvalidation method; the sensitivity and the specificity were about $96 \%$. Chincarini et al. [4] sampled the brain with seven relatively small volumes that were filtered to give intensity and textural MRI-based features. Each filtered region was analyzed with a random forest classifier to extract relevant features, which were subsequently processed with a support vector machine. The system performance was evaluated on the classification of $144 \mathrm{AD}$ patients and 189 controls. Using receiver operating curve (ROC) analysis for $144 \mathrm{AD}$ patients and 189 controls, and 20 -fold cross-validation, the result was 0.97 area under curve (AUC) for discriminating the $\mathrm{AD}$ images from the normal ones, with $89 \%$ sensitivity and $94 \%$ specificity. Chen et al. [5] used large-scale network (LSN) 
analysis to classify subjects with Alzheimer's disease and normal subjects. The Pearson product moment correlation coefficients were used to assess the connectivity between ROI in the brain. Classification of the AD group (20 subjects) and the non- $\mathrm{AD}$ group (20 subjects) using leave-one-out crossvalidation led to $87 \%$ AUC, $85 \%$ sensitivity, and $80 \%$ specificity. Graña et al. [6] used fractional anisotropy of diffusion tensor imaging (DTI) data to obtain information about the magnitude of the water diffusion process at each voxel. Based on leave-one-out cross-validation method, the linear kernel SVM achieved overall accuracy of $100 \%$ for the classification of 25 healthy controls and $20 \mathrm{AD}$ images. Wolz et al. [7] used the hippocampal volume (HV), cortical thickness $(\mathrm{CTH})$, tensor-based morphometry (TBM), and features extracted from manifold-based learning (MBL) framework to discriminate healthy controls (231 subjects) from subjects with $\mathrm{AD}$ (198 subjects). Five percent of the evaluation subjects were kept for testing and the remaining 95\% were used for training. The SVM achieved $86 \%$ correct classification rate, $94 \%$ sensitivity, and $78 \%$ specificity. Zhang et al. [8] proposed a multimodal data fusion and classification method based on features extracted from structural MRI, functional imaging (FDG-PET), and cerebrospinal fluid (CSF). The three modalities were, respectively, used to measure brain atrophy and to quantify hypometabolism and specific proteins linked to $\mathrm{AD}$. Consequently, 93 volumetric features were extracted from 93 ROI automatically labeled by an atlas warping algorithm. The linear SVM was used to evaluate the classification accuracy using 10 -fold cross-validation. The result of classifying $51 \mathrm{AD}$ and 52 normal controls yielded classification accuracy of $93.2 \%$, with a sensitivity of $93 \%$ and a specificity of $93.3 \%$. Daliri [9] used the scale-invariant feature transforms (SIFT) to extract features from MR images that were clustered using the $K$-means algorithm. Fisher's discriminant ratio was used for ranking clustered features, and genetic algorithms performed feature subset selection. The validation data consisted of the MRIs from 98 normal subjects and from 100 subjects with AD. The SVM achieved $86 \%$ correct classification rate using leave-one-out crossvalidation method. Gray et al. [10] combined cross-sectional and longitudinal FDG-PET information for classification. Particularly, the whole brain was segmented into 83 anatomically defined regions from which intensities and changes in signal intensity over the follow-up period were extracted. The SVM achieved a classification accuracy of $88 \%$ for 200 patients with Alzheimer's disease and 200 healthy controls using fivefold cross-validation protocol. Li et al. [11] used longitudinal changes of cortical thickness to characterize $\mathrm{AD}$ pathology. In particular, three categories of features were extracted from each subject, including static cortical thickness, cortex thinning dynamics, and the correlation between the longitudinal thickness changes of different regions of interest (ROI) in brain image. Based on leave-one-out crossvalidation method, the SVM distinguished $37 \mathrm{AD}$ patients from $40 \mathrm{NC}$ with an accuracy of $96.1 \%$.

In a recent work, we proposed a fractal-based processing methodology to detect AD in brain MRI [12]. The system does not require image reduction or segmentation, and it relies on a simple three-step algorithm. First, the brain MRI is transformed into a one-dimensional (1D) signal by row concatenation. Then, a three-component feature vector is extracted from the 1D signal to characterize its local and global fractal features as expressed by Hurst's exponent and the two results from the detrended fluctuation analysis (DFA) [13] of the cumulated 1D signal: the scaling exponent and the total detrended fluctuation energy (Hurst's exponent allows the evaluation of how a signal is self-affine, i.e., can be made self-similar by an affine transformation for a given level of detail (a self-similar image is one whose whole is similar to its parts); DFA is a generalization that can also detect longrange power-law correlations in seemingly nonstationary signals [13]. In particular, it can determine local trends in the signal and measure its level of persistence). In the last step, the obtained feature vector is classified by a SVM with polynomial kernel. The validation with 10 normal brain MRI and 13 corresponding to AD led to $100 \%$ classification accuracy by a SVM with quadratic kernel. The obtained results suggested that the use of fractals to characterize the MRI of normal patients and ones with $\mathrm{AD}$ held the promise of equal or better classification accuracy than the best alternative approach while being simpler to implement.

The perfect classification accuracy reported in [12] was encouraging. However, it was achieved with a small set of MRI for validation. In addition, the average processing time to extract the fractal features took over 400 seconds. This paper follows up the work in [12] with a faster algorithm and a bigger database for validation. First, the features extraction processing time is reduced considerably by using multiscale analysis (MSA) [14] as alternative for Hurst's and DFA exponent estimation. Indeed, the approach reported by Lahmiri and Boukadoum in [12] determines the sought exponents by running polynomial regressions on multiple boxes or intervals of the 1D signal; on the other hand, MSA uses the generalized Hurst's exponent method [14], which determines the scaling properties of the signal by computing the qth-order moments of the distribution of the signal's increments [14]. This method combines the sensitivity to any type of signal dependence with a high computational efficiency due to its simple algorithm [14]. As each scale $q$ allows the estimation of a Hurst's exponent, varying this value allows obtaining the multifractals of the signal in a straightforward way.

An additional contribution of this work is the use of a relatively larger database for validation in comparison to [12]. Finally, our approach does not require a predetermined region of interest as done in $[1,5,8,10]$ and avoids extracting a large number of features to characterize the image in different modalities as done in $[3,4,6-9,11]$. Instead, the multifractal analysis is performed on the whole brain MR image, and the extracted fractals are used to distinguish $\mathrm{AD}$ images from normal ones by the support vector machine. The SVM is a pattern recognition technique based on the statistical learning theory that finds the optimal nonlinear hyperplane that minimizes the expected classification error [15]. It was successfully applied in previous works $[1,3,6,8-12]$.

The balance of this paper is as follows. Section 2 presents the multiscale analysis used to obtain the generalized Hurst's exponents used to characterize brain MR images and 
the SVM used for classification. In Section 3, we describe the obtained classification performance in terms of the accuracy rate, sensibility, and sensitivity. Finally, our conclusions are drawn in Section 4.

\section{Material and Methods}

2.1. Image Database. A collection of 93 axial, T2-weighted MR brain images of $256 \times 256$ size were downloaded from the Harvard Medical School webpage [16]. The set included 51 images of normal (healthy) brains and 42 of abnormal (unhealthy) brains affected by $\mathrm{AD}$. We notice that there is no indication in the database regarding the $\mathrm{AD}$ stage. Figure 1 shows examples of healthy and AD images used in the experiments.

2.2. Multiscaling Analysis. Consider a signal $S(t)$ defined at discrete time intervals $t=v, 2 v, \ldots, T$ over a period $T$ that is an integer multiple of $v$. The qth-order moments of the distribution that characterize the statistical evolution of $S(t)$ are defined as follows [17]:

$$
K_{q}(d)=\frac{\left\langle\|S(t+d)-S(t)\|^{q}\right\rangle}{\left\langle|S(t)|^{q}\right\rangle},
$$

where $d \in\left[v, d_{\text {max }}\right]$ is a time interval and $d_{\max }$ is its predetermined upper limit. The generalized Hurst's exponent $H(q)$ is defined from the scaling behavior of $K_{q}(d)$ according to the following empirical relation [17]:

$$
K_{q}(d) \propto\left(\frac{d}{v}\right)^{q H(q)} .
$$

If $K_{q}(d)$ and $d$ satisfy a linear relationship for a given order $q$ in log-log scale, Hurst's exponent $H(q)$ can be estimated by running a linear regression of $\log \left(K_{q}(d)\right)$ versus $\log (d)$. The generalized Hurst's exponent $H(q)$ describes the longmemory dependence or persistence in the signal $S(t)$. The multiscaling structure of signal $S(t)$ is related to different orders $q$ of the exponent $H(q)$. In general, when $H(q)>0.5$, the signal fluctuations related to the order $q$ are persistent. When $H(q)<0.5$, the signal fluctuations related to order $q$ are antipersistent. Finally, the signal fluctuations are those of a random walk if $H(q)=0.5$ [17]. Notice that $H(q=2)$ corresponds to the classic Hurst's exponent [14].

In this paper, the range of $q$ is arbitrarily fixed to the interval from 1 to 6 . Higher moments could also have been considered as will be discussed in Section 4.

The original MRI is transformed into a one-dimensional (1D) signal by row concatenation. Then, Hurst's exponents $H(q)$ for $q=1, \ldots, 6$ are estimated by applying the MSA algorithm. The resulting six-component feature vector forms the input of the SVM classifier to perform the identification of $\mathrm{AD}$ images.

2.3. The Support Vector Machine Classifier. Introduced by Vapnik [15], the support vector machine (SVM) classifier is based on statistical learning theory. It implements the principle of structural risk minimization and has excellent generalization ability as a result, even when the data sample is small. The SVM performs a classification tasks by constructing an optimal separating hyperplane that maximizes the margin between the two nearest data points belonging to two separate classes. Given a training set $\left\{\left(x_{i}, y_{i}\right), i=\right.$ $1,2, \ldots, m\}$, where the input $x_{i} \in R^{d}$ and class labels $y_{i} \in$ $\{+1,-1\}$, the separation hyperplane for a linearly separable binary classification problem is given by

$$
f(x)=\langle w \cdot x\rangle+b,
$$

where $w$ is a weight vector and $b$ is a bias. The optimal separation hyperplane is found by solving the following optimization problem:

$$
\underset{w, b, \xi}{\operatorname{Minimize}} \frac{1}{2}\langle w \cdot w\rangle+C \sum_{i=1}^{m} \xi_{i}
$$

subject to

$$
y_{i}\left(\left\langle w \cdot x_{i}\right\rangle+b\right)+\xi_{i}-1 \geq 0, \quad \xi_{i} \geq 0,
$$

where $C$ is a penalty parameter that controls the tradeoff between the complexity of the decision function and the number of misclassified training examples and $\xi$ is a positive slack variable. The previous optimization model can be solved by introducing Lagrange multipliers and using the KarushKuhn-Tucker theorem of optimization to obtain the solution as

$$
w=\sum_{i=1}^{m} \alpha_{i} y_{i} x_{i}
$$

The $x_{i}$ values corresponding to positive Lagrange multipliers $\alpha_{i}$ are called support vectors, and they define the decision boundary. The $x_{i}$ values corresponding to zero $\alpha_{i}$ are irrelevant. Once the optimal values of $\alpha, \alpha^{*}$ are found, the optimal hyperplane parameters $w^{*}$ and $b^{*}$ are determined. Then, the discriminant function of the SVM for a linearly separable binary classification problem is given by

$$
g(x)=\operatorname{sign}\left(\sum_{i=1}^{m} y_{i} \alpha_{i}^{*}\left\langle x_{i} \cdot x\right\rangle+b^{*}\right) .
$$

In the nonlinearly separable case, the SVM classifier nonlinearly maps the training points to a high dimensional feature space using a kernel function $\Phi$, where linear separation can be possible. The scalar product $\left\langle\Phi\left(x_{i}\right) \cdot \Phi\left(x_{j}\right)\right\rangle$ is computed by Mercer kernel function $K$ as $K\left(x_{i}, x_{j}\right)=\left\langle\Phi\left(x_{i}\right) \cdot \Phi\left(x_{j}\right)\right\rangle$. Then, the nonlinear SVM classifier has the following form:

$$
g(x)=\operatorname{sign}\left(\sum_{i=1}^{m} y_{i} \alpha_{i}^{*} K\left\langle x, x_{i}\right\rangle+b^{*}\right) .
$$

In this study, a polynomial kernel of degree 2 was used for the SVM. As a global kernel, it allows data points that are far away from each other to also have an influence on the kernel values. The general polynomial kernel is given by

$$
K\left(x, x_{i}\right)=\left(\left(x_{i} \cdot x\right)+1\right)^{d}
$$




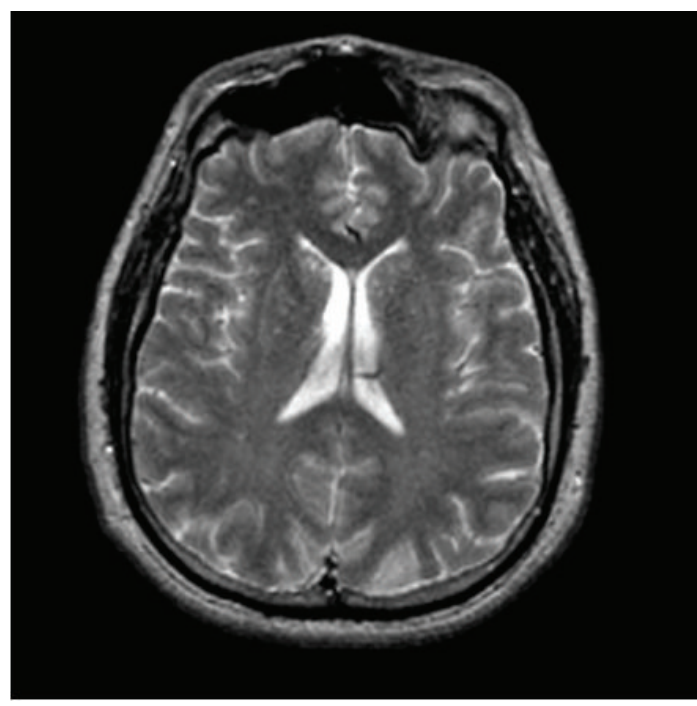

(a)

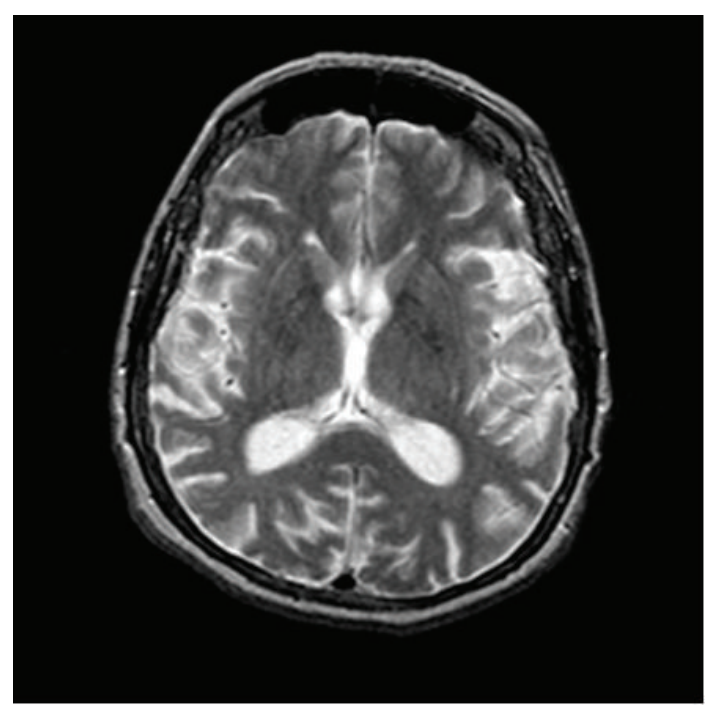

(b)

FIGURE 1: Healthy image (a) and AD image (b) in grayscale.

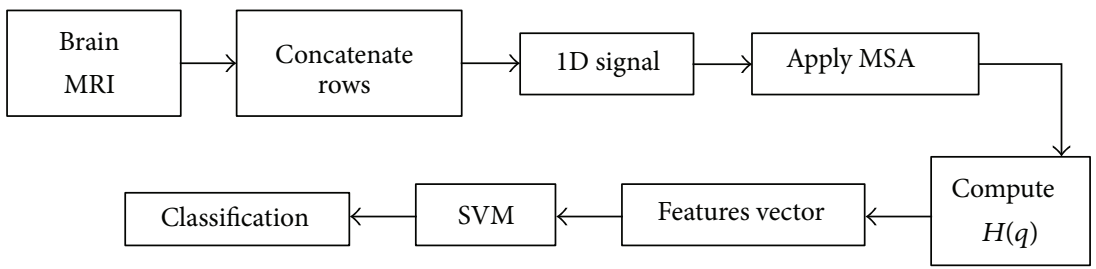

FIgURE 2: AD diagnosis system.

where $d$ is the order of the polynomial to be used. In this study, it was varied from 2 to 4 . Higher orders were ignored because of a higher computational burden with no substantial gain in classification accuracy from our experience.

2.4. Validation. The design of the automated AD diagnosis system is shown in Figure 2.

The validation experiments were conducted using the leave-one-out cross-validation method. Then, the average and standard deviation of the correct classification rate (CCR), sensitivity, and specificity were computed to evaluate the performance of the classifier. The three performance measures are defined as follows:

$$
\begin{gathered}
\text { CCR }=\frac{\text { Classified Samples }}{\text { Total Number of Samples }}, \\
\text { Sensitivity }=\frac{\text { Correctly Classified Positive Samples }}{\text { True Positive Samples }}, \\
\text { Specificity }=\frac{\text { Correctly Classified Negative Samples }}{\text { True Negative Samples }},
\end{gathered}
$$

where positive samples and negative samples refer to $\mathrm{AD}$ and normal images, respectively.

\section{Results}

Before processing, the grayscale images as shown in Figure 1 are converted to double color format to perform MSA (see examples in Figure 3). Figure 4 shows the obtained behavior of $K_{q}(d)$ as a function of $d$ (see, (2)) for the images of healthy patients and those with $\mathrm{AD}$ and for $q$ varying from 1 to 6 , whereas $d$ varies from 1 to 19 . Figure 5 provides the same information on a log-log scale. Figure 4 reveals that, for the images of normal brains, $K_{q}(d)$ quickly reaches horizontal saturation for $q=1,2,3$; this is not so for the AD images where $K_{q}(d)$ increases monotonically for all $d$. In addition, the magnitude of $K_{q}(d)$ for a healthy image is in general larger than that of an $\mathrm{AD}$ image. In summary, Hurst's exponents appear to be different at each scale for the two types of brain MRIs.

As mentioned previously, the order of the polynomial kernel used in the SVM was varied from 2 to 4 . The best classification performance was obtained with a fourth-order kernel, for which the correct classification rate, sensitivity, and specificity were $99.18 \% \pm 0.0083,100 \%$, and $98.20 \% \pm$ 0.0182 , respectively. A visual analysis of the $\mathrm{AD}$ images revealed that seventeen of them looked markedly different from the example in Figure 1 (Figure 6 shows three of them). Removing them from the validation database led to $100 \%$ classification accuracy by the SVM. 


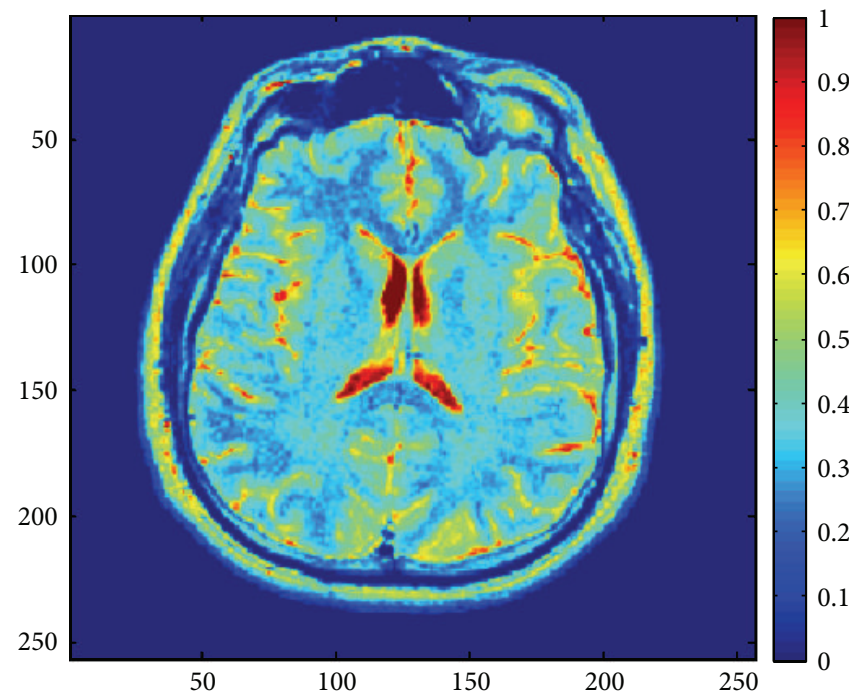

(a)

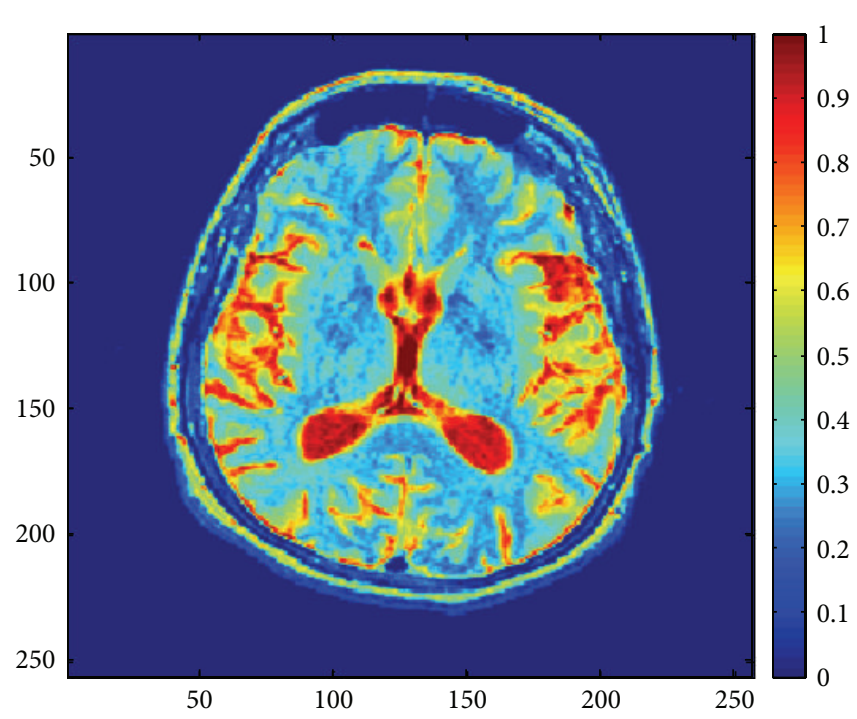

(b)

FIgURe 3: Healthy image (a) and AD image (b) in double color format.

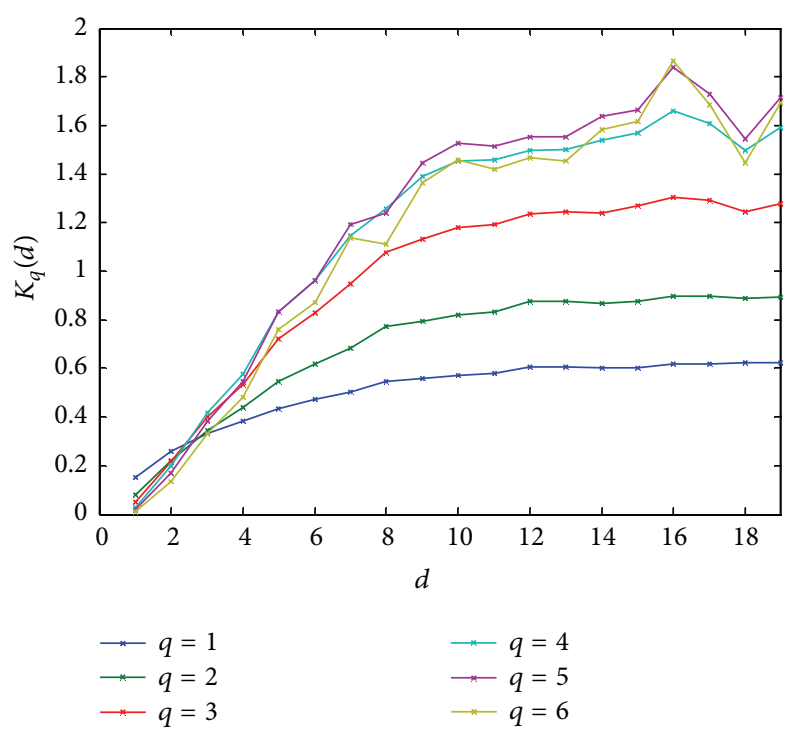

(a)

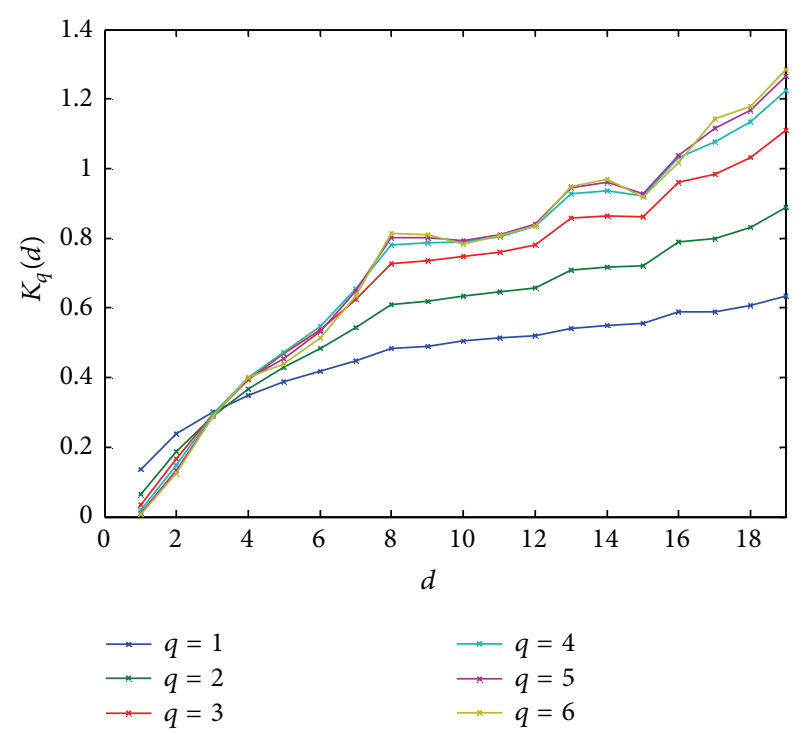

(b)

FIGURE 4: Multiscale analysis results of healthy image (a) and AD (b).

Finally, the MSA running time was 5.64 seconds, and the overall image processing time was about 8 seconds on a $3.30 \mathrm{GHz}$ Core i5-2500 CPU using MATLAB 2012a codes, leading to an execution speed improvement of nearly two orders of magnitude in comparison to Hurst's exponent and DFA.

\section{Discussion and Conclusion}

The overall classification accuracy obtained in this work was $99.18 \% \pm 0.01$. However, excluding some atypical AD images led to $100 \%$ correct classification rate. This suggests that a multi-SVM approach, where one or more classifier handles the misclassified images by the first SVM, might lead to perfect classification accuracy in all situations. We intend to investigate this possibility.

Our approach for $\mathrm{AD}$ detection outperformed most of the studies found in the literature, where the reported classification accuracy was between $86 \%$ and $96.9 \%[1-5,7-13]$. One exception is the work of [6] where perfect classification accuracy is reported. However, it is achieved with a database on only $45 \mathrm{MR}$ images ( 25 healthy controls and $20 \mathrm{AD}$ images), and the number of features used was higher than 1000 in comparison to the six used by our approach. We limited the number of features to six somewhat arbitrarily, and we have not investigated the effect of more or less features 


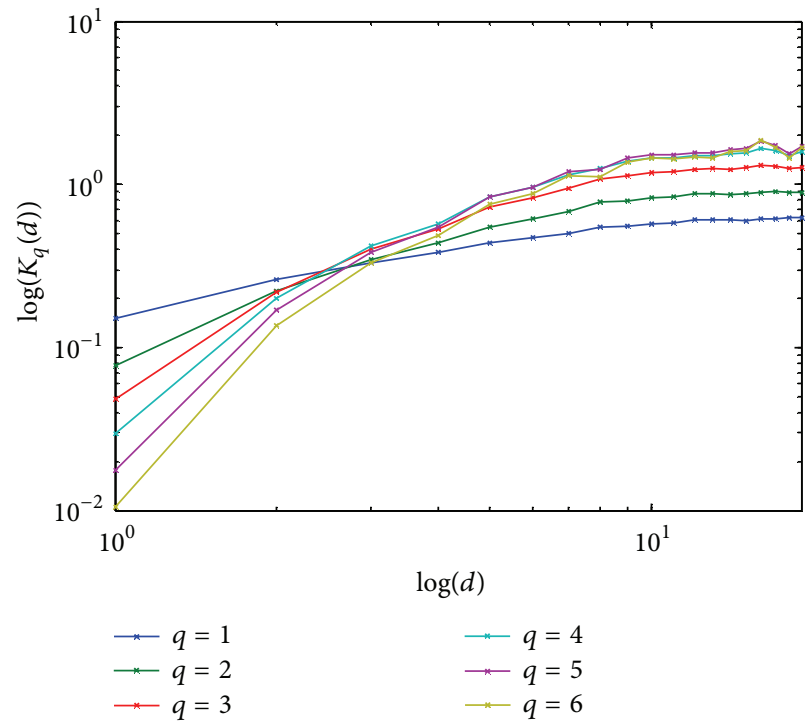

(a)

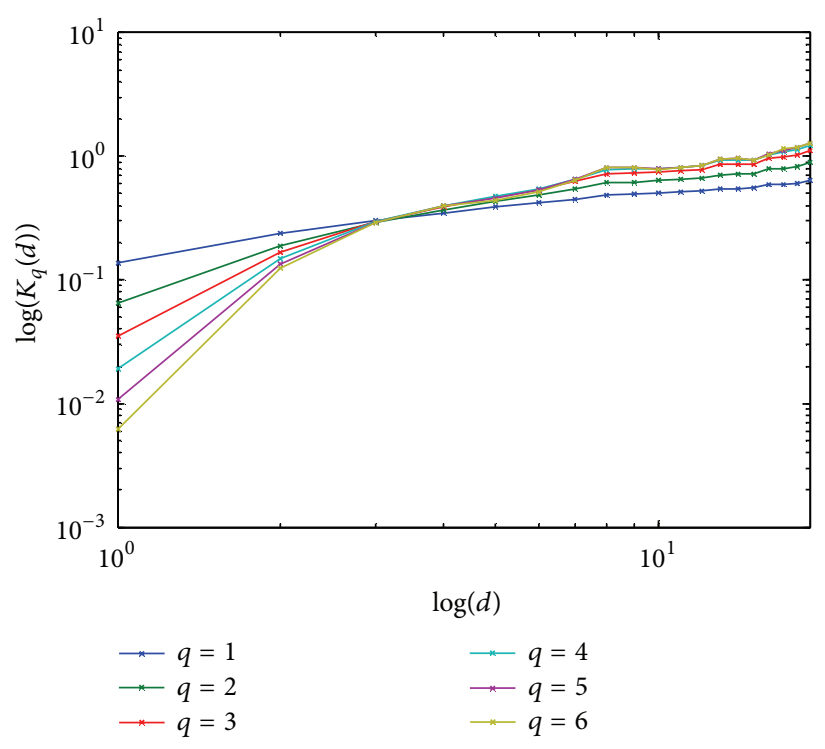

(b)

FIGURE 5: Multiscale analysis results on log-log scale of healthy image (a) and AD (b).
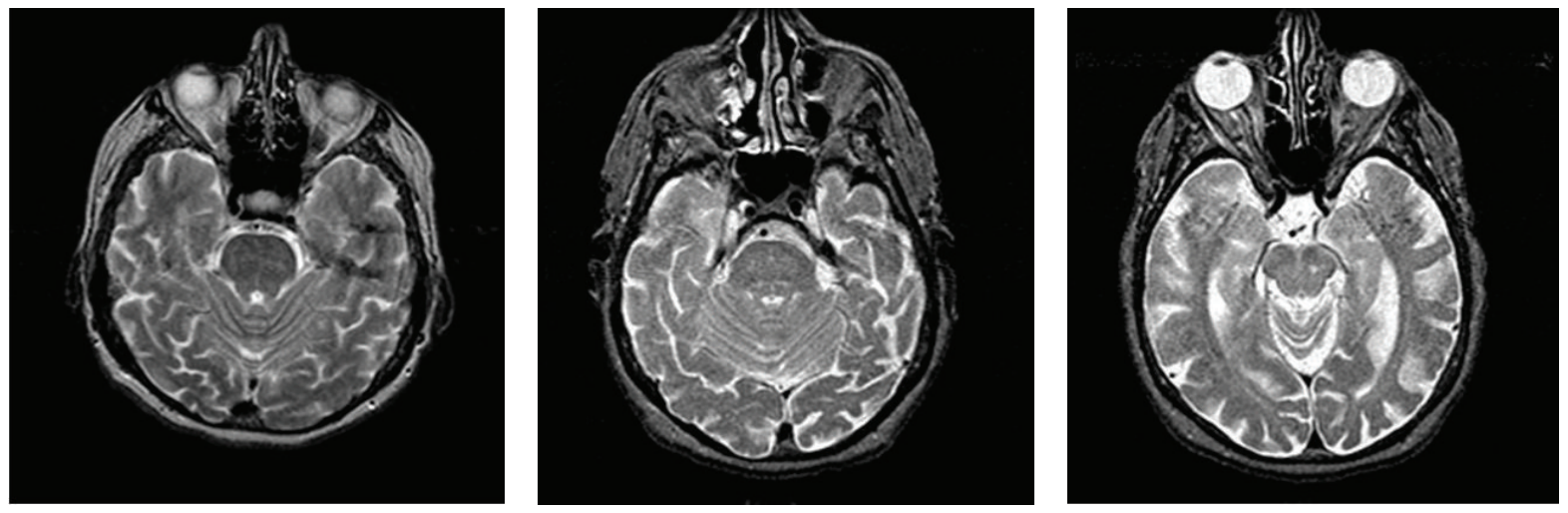

FIgURE 6: Examples of excluded AD images in the second experiment.

on the performance of the classifier. Future work should also investigate this.

In summary, multiscale analysis-based Hurst's exponents were used for the classification of healthy brain images versus AD by a SVM with fourth-order kernel. The obtained results show the potential of using multiscale fractal analysis to differentiate healthy brain images from ones affected by Alzheimer's disease. The MSA algorithm took 5.64 seconds to analyze a brain MRI while the detrended fluctuation analysis (DFA) took 400 seconds in our previous work [12]. In addition, a larger database is used for validation.

Finally, although we have obtained better result than the literature in general, it is difficult to draw definite conclusions since we used a different image database. In future work, we will explore a benchmark image depository such as the Alzheimer's Disease Neuroimaging Initiative (ADNI) database. Furthermore, we will investigate the effectiveness of MSA to classify AD images versus mild cognitive impairment (MCI). Indeed, the ability to correctly classify the $\mathrm{AD}$ and MCI images based on MSA Hurst's exponents might shed light on the ability to predict the conversion from MCI to AD, which is of clinical interest.

\section{References}

[1] B. Magnin, L. Mesrob, S. Kinkingnéhun et al., "Support vector machine-based classification of Alzheimer's disease from whole-brain anatomical MRI," Neuroradiology, vol. 51, no. 2, pp. 73-83, 2009.

[2] J. Ramírez, J. M. Górriz, F. Segovia et al., "Computer aided diagnosis system for the Alzheimer's disease based on partial least squares and random forest SPECT image classification," Neuroscience Letters, vol. 472, no. 2, pp. 99-103, 2010.

[3] D. Salas-Gonzalez, J. M. Górriz, J. Ramírez et al., "Computeraided diagnosis of Alzheimer's disease using support vector machines and classification trees," Physics in Medicine and Biology, vol. 55, no. 10, pp. 2807-2817, 2010.

[4] A. Chincarini, P. Bosco, P. Calvini et al., "Local MRI analysis approach in the diagnosis of early and prodromal Alzheimer's disease," NeuroImage, vol. 58, no. 2, pp. 469-480, 2011. 
[5] G. Chen, B. D. Ward, C. Xie et al., "Classification of Alzheimer disease, mild cognitive impairment, and normal cognitive status with large-scale network analysis based on resting-state functional MR imaging," Radiology, vol. 259, no. 1, pp. 213-221, 2011.

[6] M. Graña, M. Termenon, A. Savio et al., "Computer aided diagnosis system for Alzheimer disease using brain diffusion tensor imaging features selected by Pearson's correlation," Neuroscience Letters, vol. 502, no. 3, pp. 225-229, 2011.

[7] R. Wolz, V. Julkunen, J. Koikkalainen et al., "Multi-method analysis of MRI images in early diagnostics of Alzheimer's disease," Plos One, vol. 6, no. 10, Article ID e25446, 2011.

[8] D. Zhang, Y. Wang, L. Zhou, H. Yuan, and D. Shen, "Multimodal classification of Alzheimer's disease and mild cognitive impairment," NeuroImage, vol. 55, no. 3, pp. 856-867, 2011.

[9] M. R. Daliri, "Automated diagnosis of Alzheimer disease using the scale-invariant feature transforms in magnetic resonance images," Journal of Medical Systems, vol. 36, no. 2, pp. 995-1000, 2012.

[10] K. R. Gray, R. Wolz, R. A. Heckemann, P. Aljabar, A. Hammers, and D. Rueckert, "Multi-region analysis of longitudinal FDGPET for the classification of Alzheimer's disease," NeuroImage, vol. 60, no. 1, pp. 221-229, 2012.

[11] Y. Li, Y. Wang, G. Wu et al., "Discriminant analysis of longitudinal cortical thickness changes in Alzheimer's disease using dynamic and network features," Neurobiology of Aging, vol. 33, no. 2, pp. 427.e15-427.e30, 2012.

[12] S. Lahmiri and M. Boukadoum, "Automatic detection of Alzheimer disease in brain magnetic resonance images using fractal features," in Proceedings of the 6th International IEEE EMBS Conference on Neural Engineering, 2013.

[13] H. E. Stanley, L. A. N. Amaral, A. L. Goldberger, S. Havlin, P. C. Ivanov, and C.-K. Peng, "Statistical physics and physiology: monofractal and multifractal approaches," Physica A, vol. 270, no. 1, pp. 309-324, 1999.

[14] T. Di Matteo, "Multi-scaling in finance," Quantitative Finance, vol. 7, no. 1, pp. 21-36, 2007.

[15] V. Vapnik, The Nature of Statistical Learning Theory, Springer, Berlin, Germany, 1995.

[16] http://www.med.harvard.edu/AANLIB/.

[17] C. K. Peng, S. V. Buldyrev, S. Havlin, M. Simons, H. E. Stanley, and A. L. Goldberger, "Mosaic organization of DNA nucleotides," Physical Review E, vol. 49, no. 2, pp. 1685-1689, 1994. 


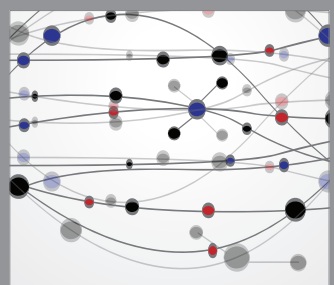

The Scientific World Journal
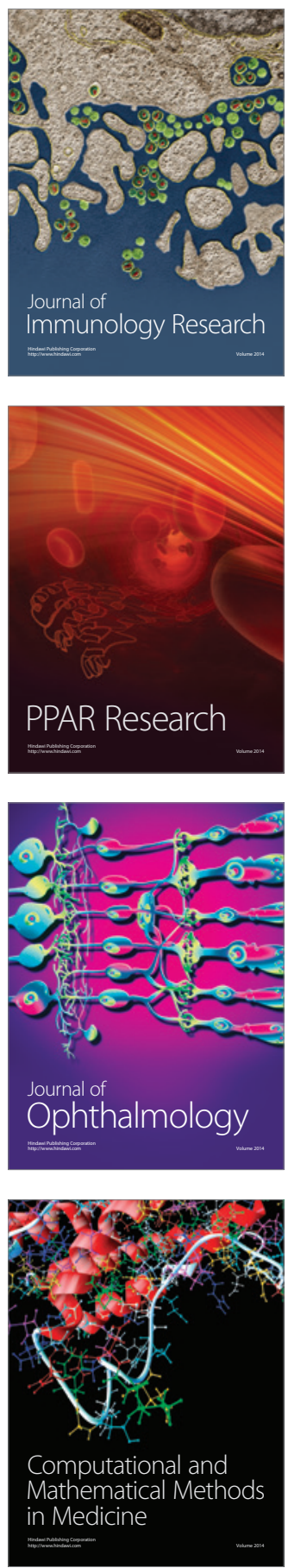

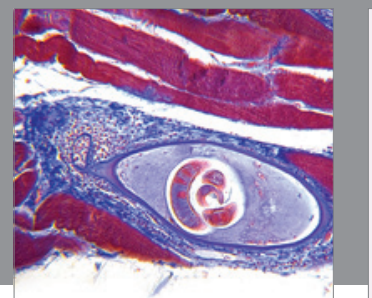

Gastroenterology

Research and Practice
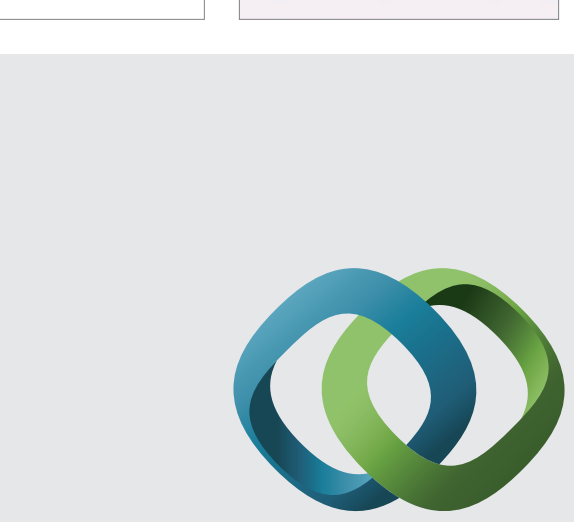

\section{Hindawi}

Submit your manuscripts at

http://www.hindawi.com
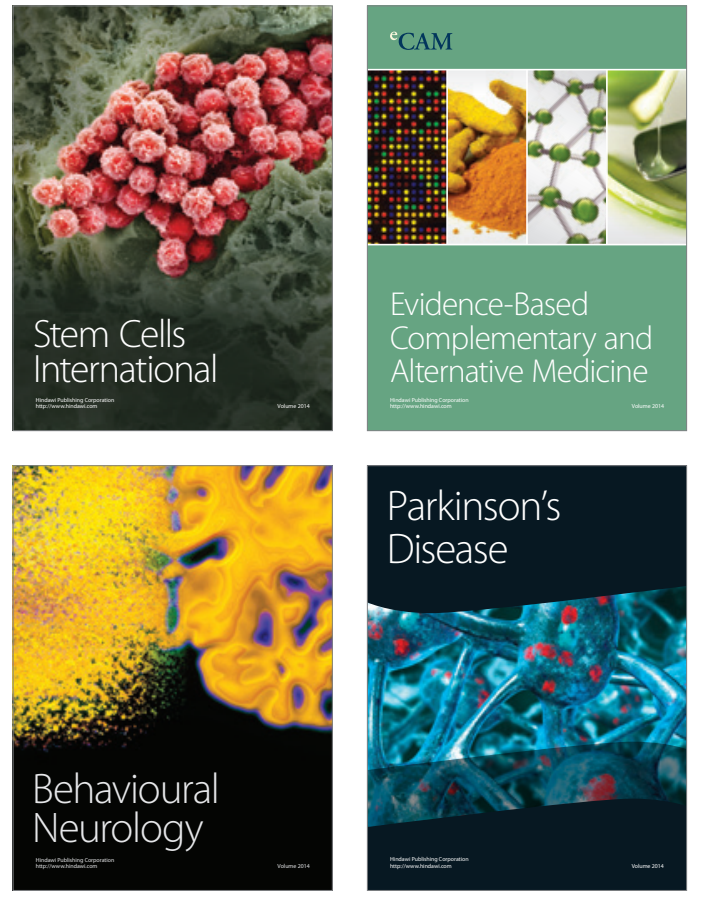
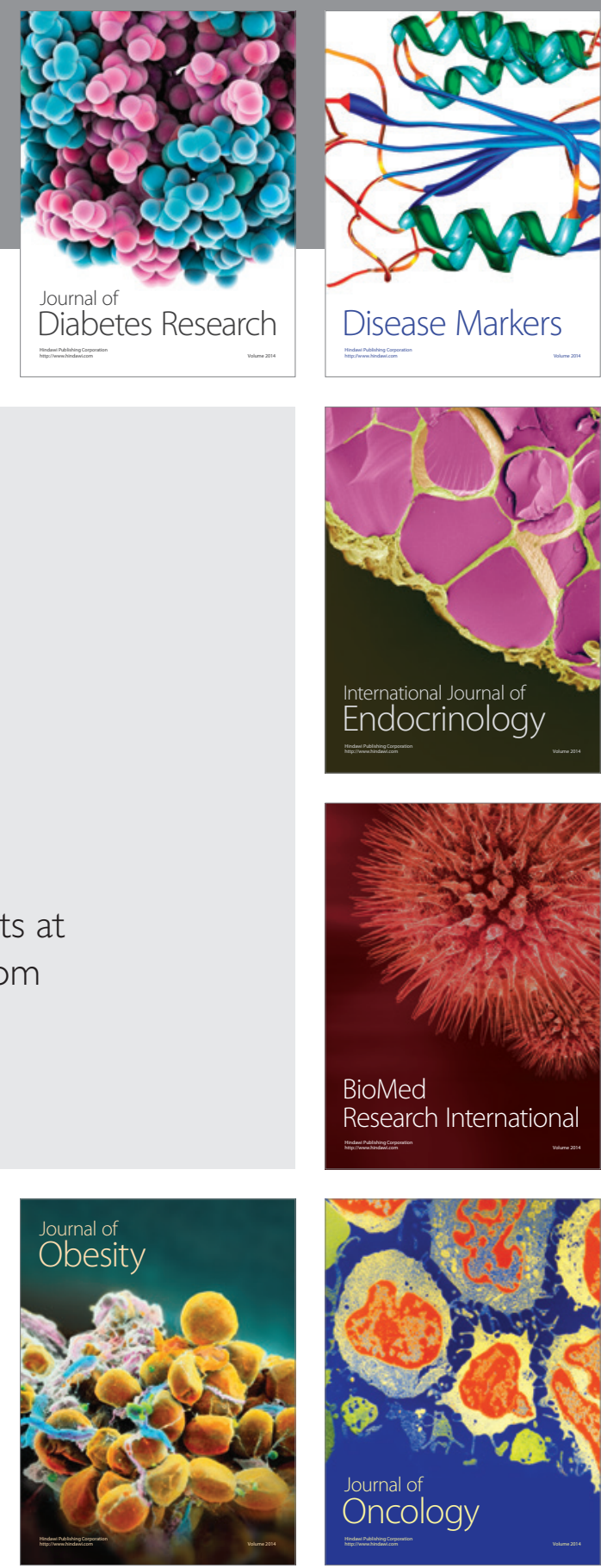

Disease Markers
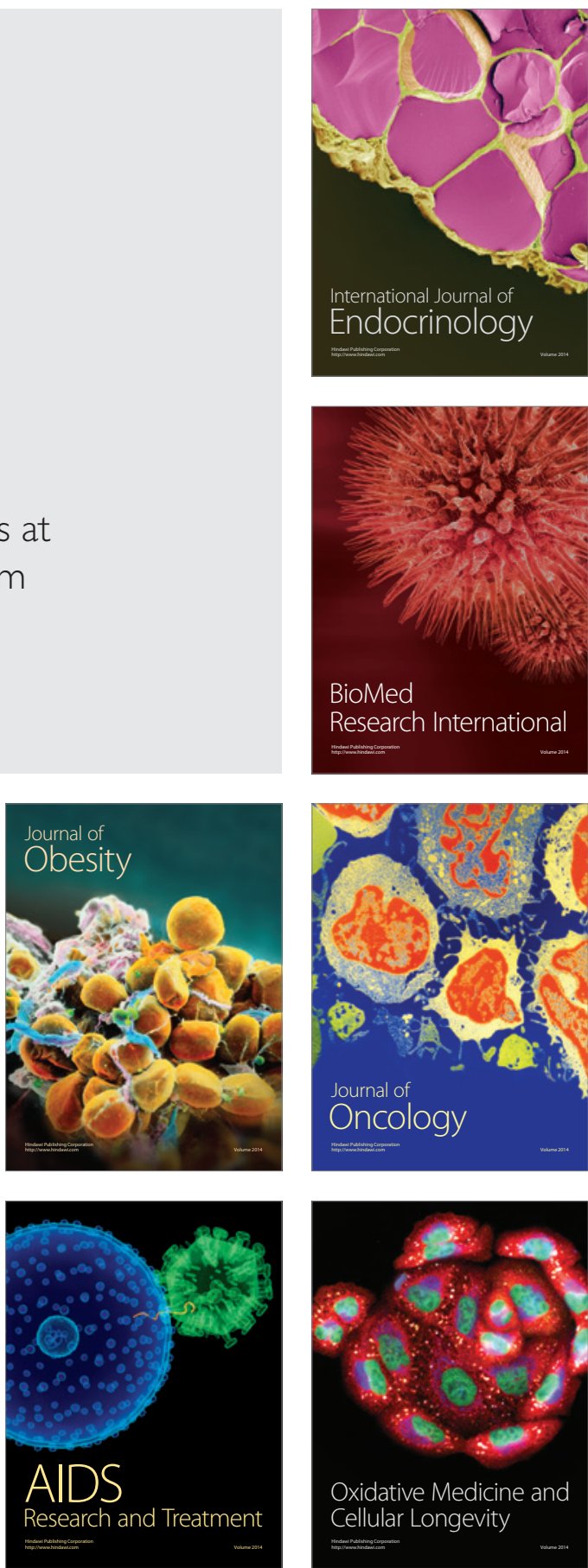